SOI: $\underline{1.1 / \mathrm{TAS}} \quad$ DOI: $\underline{10.15863 / \mathrm{TAS}}$
International Scientific Journal
Theoretical \& Applied
Science
p-ISSN: $2308-4944$ (print) $\quad$ e-ISSN: $2409-0085$ (online)
Year: $2016 \quad$ Issue: $8 \quad$ Volume: 40
Published: $30.08 .2016 \quad$ http://T-Science.org

SECTION 7. Mechanics and machine construction.

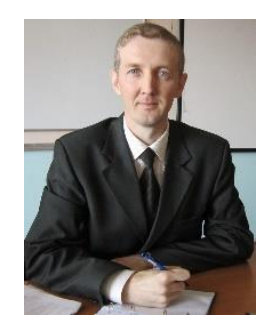

Almir Anfirovich Miniakhmetov Headmaster's assistant, Teacher of Physics, Computer Science and Information Technologies Askino Secondary School №1, Republic of Bashkortostan, Russian Federation minalmir@yandex.ru

Salavat Aidarovich Abdullin The 4th years Student of Electrical Engineering, Faculty Avionics, Energy and Information Communications Ufa State Aviation Technical University, Ufa, Republic of Bashkortostan, Russian Federation zalavat-abdullin@mail.ru

\title{
ANALYSIS OF SOME WORKING CHARACTERISTICS OF A SINGLE- PHASE INDUCTION MOTOR WITH CANNED POLES
}

\begin{abstract}
The article considers some working characteristics of a single-phase induction motor with canned poles, it also carries out their physical analysis. Provided a generally accepted in electric engineering salient pole construction of a motor, constructed a clock diagram of residual flux of two parts of the pole, and rigged up a motor switching in a single-phase network. Suggested basic electro physical characteristic curves for motor working characteristics. Analyzed electro physical parameters of basic motor working characteristics with respect to the first space harmonic of air-gap field. Shown basic electro physical characteristic curves for motor working characteristics with respect to differential scattering of a stator winding. Recommended a method of improving and development of control characteristics for the decrease of energy losses and the increase of the efficiency of a single-phase motor with canned poles.
\end{abstract}

Key words: induction motor, alternating current, single-phase, salient pole, canned, efficiency, control characteristics, equivalent system of substitution.

Language: Russian

Citation: Miniakhmetov AA, Abdullin SA (2016) ANALYSIS OF SOME WORKING CHARACTERISTICS OF A SINGLE-PHASE INDUCTION MOTOR WITH CANNED POLES. ISJ Theoretical \& Applied Science, 08 (40): $1-7$.

Soi: http://s-0-i.org/1.1/TAS-08-40-1 Doi: crossef http://dx.doi.org/10.15863/TAS.2016.08.40.1

\section{УДК 621.31}

\section{АНАЛИЗ НЕКОТОРЫХ РАБОЧИХ ХАРАКТЕРИСТИК ОДНОФАЗНОГО АСИНХРОННОГО ДВИГАТЕЛЯ С ЭКРАНИРОВАННЫМИ ПОЛЮСАМИ}

Аннотация: В статье рассмотрены некоторые рабочие характеристики однофазного асинхронного двигателя с экранированными полюсами, а также проведен их физический анализ. Приведена общепринятая в электротехнике схема явнополюсной конструкиии двигателя, построена векторная диаграмма магнитных потоков двух частей полюса, а также собрана электрическая схема включения двигателя в однофазную сеть. Предложены основные электрофизические зависимости для рабочих характеристик двигателя. Проанализированы электрофизические параметры основных рабочих характеристик двигателя с учетом первой пространственной гармоники магнитного поля в воздушном зазоре. Показаны электрофизические зависимости рабочих характеристик с учетом дифференциального рассеяния статорной обмотки. Рекомендован метод улучшения и усовершенствования пусковых характеристик для уменьшения энергетических потерь и увеличения коэффициента полезного действия однофазного асинхронного двигателя с экранированными полюсами.

Ключевые слова: асинхронный двигатель, переменный ток, однофазный, явнополюсной, экранированный, коэффициент полезного действия, пусковые характеристики, эквивалентная схема замещения.

Введение. Асинхронный двигатель [1] представляет собой асинхронную машину, работающую на переменном токе, которая предназначена, в свою очередь, для 
преобразования электрической энергии переменного тока в механическую энергию.

Понятие "асинхронныци" с точки зрения физики означает, что у такого рода двигателей частота вращения магнитных полей статора $n_{1}$ и частота вращения магнитных полей ротора $n_{2}$ не совпадают.

На сегодняшний день подобные двигатели получили довольно широкое применение благодаря высокой надежности, низкой стоимости и простой конструкции. Согласно недавним исследованиям [2], такие двигатели составляют порядка $90 \%$ от общего числа двигателей в мире. Можно даже сказать, что из-за изобретения асинхронных двигателей и произошел большой переворот в промышленности. Огромную популярность они получили благодаря простоте эксплуатации и дешевизне конструкции.

В последние годы [3, 4] все чаще наблюдается заметный рост интереса к электродвигателям переменного тока как большой, так и малой мощности. Это объясняется следующими причинами: (1) Простота и технологичность конструкции подобных машин; (2) Высокая надежность; (3) Расширяющаяся область применения подобных машин в быту и промышленности.

Однако, основную долю среди микроэлектродвигателей переменного тока занимают однофазные явнополюсные асинхронные двигатели [5]. Одним из типичных представителей машин этого класса являются асинхронные электродвигатели с экранированными полюсами [6].

Тем не менее, до сих пор ведутся расчеты по улучшению рабочих характеристик для более эффективного использования указанного электродвигателя.
Постановка цели. В связи с этим, целью данной работы является анализ некоторых рабочих характеристик однофазного асинхронного двигателя и возможные пути совершенствования конструкции для повышения коэффициента полезного действия и уменьшения потери.

Актуальность статьи заключается в том, что проведен анализ некоторых технических параметров однофазных асинхронных двигателей с целью совершенствования конструкции и повышения технико-экономических показателей.

Научная новизна исследования состоит в том, что проведен анализ основных рабочих характеристик, энергетических показателей двигателя с целью улучшения пусковых характеристик, а также основные зависимости для их практических расчетов.

Краткая теория. Однофазные асинхронные двигатели с экранированными полюсами являются самыми распространенными однофазными электродвигателями малой мощности. Они характеризуются не только высокой надежностью, но и бесконтактностью и отсутствием радиопомех, способностью работать в повторно-коротковременном режиме, относительно низкой стоимостью, возможностью работать в односкоростном и многоскоростном режимах.

Согласно [7], статор подобного двигателя имеет явнополюсную конструкцию (рис. 1).

Видно, что на полюсах размещается однофазная обмотка, подключаемая в однофазную сеть. Часть полюса охватывает короткозамкнутый виток $K$. На роторе двигателя имеется обыкновенная короткозамкнутая обмотка в виде беличьей клетки.

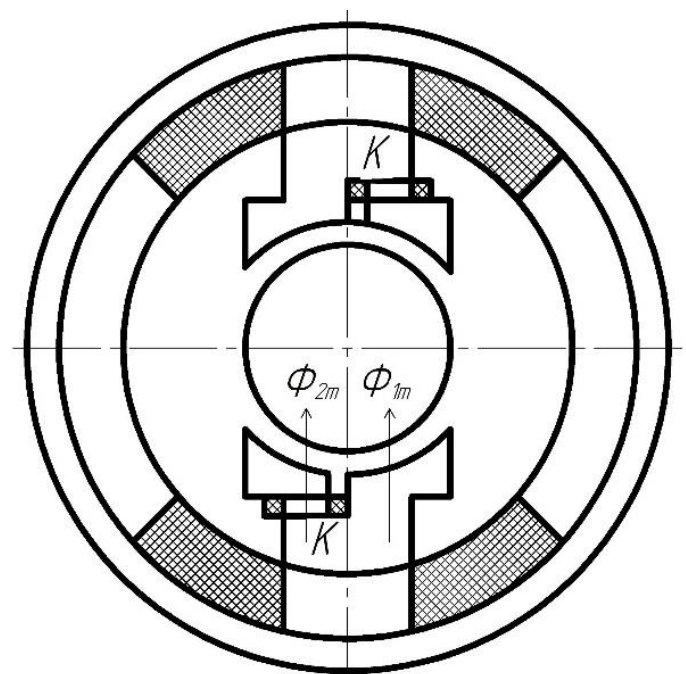

Рисунок 1 - Схематическая конструкция статора и ротора однофазного асинхронного двигателя с экранированными полюсами.

Магнитный поток одного полюса двигателя можно представить в виде двух составляющих. 
Часть полюса, не охваченную короткозамкнутым витком, пронизывает магнитный поток $\Phi_{1 \mathrm{~m}}$, создаваемый током обмотки статора $I_{1}$.

Другую часть полюса можно рассматривать как трансформатор, у которого первичной обмоткой является обмотка самого статора, а вторичной - короткозамкнутый виток. Результирующий магнитный поток этой части полюса $\Phi_{2 \mathrm{~m}}$ будет представлять собой геометрическую сумму потока $\Phi_{2 \mathrm{~m}}^{\prime}$, созданного током обмотки статора $I_{1}$, и магнитного потока $\Phi_{\mathrm{km}}$ от тока $I_{\mathrm{k}}, \quad$ индуктируемого в короткозамкнутом витке магнитным потоком $\Phi_{2 \mathrm{~m}}^{\prime}$.

Опираясь на [8], построим векторную диаграмму (рис. 2) и проведем краткий анализ. Между магнитными потоками двух частей полюса $\Phi_{1 \mathrm{~m}} \quad$ и $\Phi_{2 \mathrm{~m}}$ из-за экранирующего действия короткозамкнутого витка, существует сдвиг по фазе во времени на угол $\beta$.

Кроме этого, оси этих магнитных потоков смещены на определенный угол в пространстве, поэтому, магнитное поле в машине будет вращающимся. Так как магнитные потоки $\Phi_{1 \mathrm{~m}}$ и $\Phi_{2 \mathrm{~m}}$ не равны между собой, а также, угол сдвига между ними меньше чем $90^{\circ}$, то магнитное поле уже будет эллиптическим.

Вследствие этого, двигатели с экранированными полюсами имеют определенные недостатки, такие как: (1) Большие габаритные размеры; (2) Небольшой пусковой момент $\left(M_{\mathrm{n}} \sim 0,2-0,5\right.$ от номинального момента); (3) Низкий коэффициент мощности $(\cos \varphi \sim 0,4$ $0,6)$; (4) Невысокий коэффициент полезного действия из-за больших потерь в короткозамкнутом витке $(\eta \sim 0,25-0,4)$.

В таких двигателях, как правило, ротор всегда вращается в направлении от неэкранированной части полюса к экранированной части полюса. Короткозамкнутый виток приводит к большим потерям мощности, поэтому коэффициент полезного действия $\eta$ двигателя составляет порядка 40\%. Большие потоки рассеяния обмотки ротора, которые замыкаются через полюсные наконечники, приводят к большим индуктивным сопротивлениям этой обмотки, в связи с чем, кратность пускового тока двигателя заметно уменьшается.

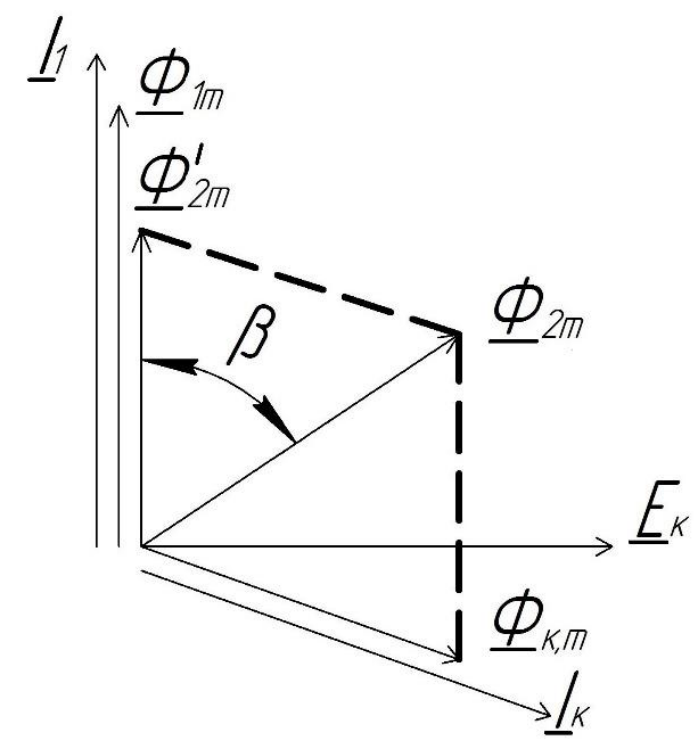

Рисунок 2 - Векторная диаграмма магнитных потоков полюсов однофазного асинхронного двигателя с экранированными полюсами.

С другой стороны, по конструктивному исполнению асинхронные двигатели с экранированными полюсами подразделяются на двигатели с несимметричным расположением главной статорной обмотки [9] и с симметричным расположением главной статорной обмотки [10].

Двигатель с несимметрично расположенной главной статорной обмоткой имеет значительное рассеяние магнитного потока во внешней магнитной цепи, вследствие чего его коэффициент полезного действия $\eta$ не превышает $10-15 \%$, и такие двигатели изготавливаются лишь до мощности $\sim 10 \mathrm{Bm}$.

С точки зрения технологичности, двигатель с симметрично расположенной главной обмоткой является более сложным. В двигателях с мощностями $\sim 10-50 \mathrm{Bm}$ используется составной статор.

Ввиду того, что полюсы двигателя охвачены ярмом, и обмотки расположены внутри магнитной системы, магнитные потоки рассеяния в этом случае, значительно меньше, чем в предыдущей 
конструкции. Коэффициент полезного действия $\eta$ двигателя составляет уже 15-25\%.

В двигателях мощностью $150 \mathrm{Bm}$ уже используют неразборную систему статора. Реверс такого двигателя возможен только в том случае, если с обеих сторон полюса расположены короткозамкнутые обмотки, замыкаемые накоротко с помощью дополнительного переключения. Однако, в этом случае приходится использовать дополнительный переключатель довольно сложной конструкции, контакты которого рассчитаны на значительный ток.

Только по этой причине предпочтительней использовать два асинхронных двигателя с экранированными полюсами, каждый из которых включается только при соответствующем направлении вращения.

В современной теории асинхронных двигателей, для расчета рабочих характеристик, применяется метод эквивалентных схем замещения $[11,12]$. Этот метод удобен тем, что схемы замещения позволяют свести сложные процессы, происходящие в электрической машине, к зависимостям между токами и напряжениями.
Это означает, например, что в последующем можно проводить анализ вольтамперных характеристик на соответствующих графиках и зависимостях [13].

Однако, применение метода схем замещения к несимметричным асинхронным машинам довольно затруднительный процесс, в связи с необходимостью учета высших пространственных гармоник, что приводит к увеличению контуров схемы замещения.

Подобные проблемы возникают при анализе вольтамперных характеристик двигателей, поэтому расчет рабочих характеристик обычно принято производить по первой (основной) пространственной гармонике магнитного поля в воздушном зазоре.

Анализ характеристик и результаты. Опираясь главным образом на [14], выявим основные зависимости для электромагнитного момента однофазного асинхронного двигателя с экранированными полюсами.

Известно, что действующее значение токов ротора прямой и обратной последовательности определяются следующими формулами:

$$
\begin{aligned}
& I_{2 v}^{\prime+}=\frac{E_{v}^{+}}{\sqrt{\left(r_{2 v}^{\prime} / s_{v}^{-}\right)^{2}+\left(x_{2 v}^{\prime}\right)^{2}}} \\
& I_{2 v}^{\prime+}=\frac{E_{v}^{-}}{\sqrt{\left(r_{2 v}^{\prime} / s_{v}^{-}\right)^{2}+\left(x_{2 v}^{\prime}\right)^{2}}} .
\end{aligned}
$$

Сдвиг по фазе между электродвижущей силой и токами ротора для магнитного поля прямой и обратной последовательности определяются равенствами:

$$
\begin{aligned}
& \psi_{2 v}^{+}=a \cos \left(\frac{r_{2 v}^{\prime} / s_{v}^{+}}{\sqrt{\left(r_{2 v}^{\prime} / s_{v}^{+}\right)^{2}+\left(x_{2 v}^{\prime}\right)^{2}}}\right) \\
& \psi_{2 v}^{-}=a \cos \left(\frac{r_{2 v}^{\prime} / s_{v}^{-}}{\sqrt{\left(r_{2 v}^{\prime} / s_{v}^{+}\right)^{2}+\left(x_{2 v}^{\prime}\right)^{2}}}\right)
\end{aligned}
$$

Зависимость для электромагнитного момента $v$-той гармоники будем иметь выражение следующего вида:

$$
M_{v}=\frac{p \cdot m_{1}}{\omega_{1}} \cdot\left(E_{v}^{+} \cdot I_{2 v}^{\prime+} \cdot \cos \left(\psi_{2 v}^{+}\right)-E_{v}^{-} \cdot I_{2 v}^{\prime-} \cdot \cos \left(\psi_{2 v}^{-}\right)\right),
$$

где $m_{1}$ представляет собой число фаз статора.
Преобразуем все описанные выше выражения (1), (2), (3), получим комбинированное выражение вида: 


$$
M_{v}\left(s_{v}^{+}, s_{v}^{-}\right)=\frac{p \cdot m_{1}}{\omega_{1}} \cdot\left(\frac{\left(E_{v}^{+}\right)^{2} \cdot r_{2 v}^{\prime} / s_{v}^{+}}{\left(r_{2 v}^{\prime} / s_{v}^{+}\right)^{2}+\left(x_{2 v}^{\prime}\right)^{2}}-\frac{\left(E_{v}^{-}\right)^{2} \cdot r_{2 v}^{\prime} / s_{v}^{-}}{\left(r_{2 v}^{\prime} / s_{v}^{-}\right)^{2}+\left(x_{2 v}^{\prime}\right)^{2}}\right)
$$

Как известно, суммарный электромагнитный момент $M$ определяется по общеизвестной формуле следующего вида:

$$
M=\sum_{v=1}^{\infty} M_{v}
$$

С другой стороны, механическая мощность $P_{\text {мх }}$ через электромагнитный момент будет представлена в виде выражения вида:

$$
P_{\mathrm{Mx}}=\left(1-s_{1}^{+}\right) \cdot \frac{\omega_{1}}{p} \cdot M
$$

Полезная мощность рассчитывается по следующему выражению:

$$
P_{2}=P_{\text {мх }}-\left(P_{\text {мех }}+P_{\text {доб }}\right),
$$

где $p_{\text {мех }}-$ механические потери, $p_{\text {доб }}-$ добавочные потери.

Для определения других энергетических показателей будем использовать эквивалентную модель двигателя, подробно описанную в [15], приведя соответствующую электрическую схему (рис. 3) включения эквивалентного асинхронного двигателя в однофазную сеть.

Реальная электрическая схема была собрана и апробирована практически на кафедре электромеханики Уфимского государственного авиационного технического университета г.Уфы Республики Башкортостан.

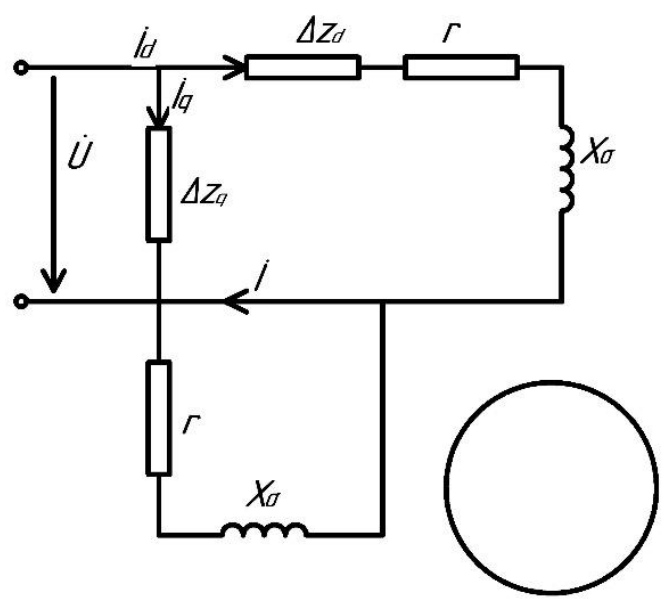

Рисунок 3 - Электрическая схема включения эквивалентного однофазного асинхронного двигателя с экранированными полюсами в однофазную сеть.

Расчет целесообразно проводить для основной гармоники поля. Уравнения эквивалентной модели, записанного в режиме короткого замыкания, имеют следующий вид:

$$
\begin{aligned}
& \dot{U}=\left(I_{1}^{+}+I_{1}^{-}\right) \cdot \Delta z_{d}+I_{1}^{+} \cdot\left(z+z_{1}\right)+I_{1}^{-} \cdot\left(z+z_{1}\right) \\
& \dot{U}=j \cdot\left(I_{1}^{+}+I_{1}^{-}\right) \cdot \Delta z_{q}+j \cdot I_{1}^{-} \cdot\left(z+z_{1}\right)-j \cdot I_{1}^{-} \cdot\left(z+z_{1}\right) .
\end{aligned}
$$

При заторможенном роторе величины $z$ и $z_{1}$ определяются симметрией машины и параметрами реального статора и ротора.

Решим последнюю систему уравнений (8) относительно неизвестных величин $\Delta z_{\mathrm{d}}$ и $\Delta z_{\mathrm{q}}$. В результате получим значения сопротивлений, которые позволяют определить временные параметры магнитного поля в воздушном зазоре эквивалентной машины, и, косвенным образом позволяют учитывать влияние высших пространственных гармоник.

Мы объясняем это следующим образом. Хотя высшие пространственные гармоники исключены из рассмотрения, нельзя не учитывать потерь энергии, связанные с ними.

В классической теории электрических машин влияние гармоник выше первой, учитывают с помощью дифференциального рассеяния статорной обмотки [16].

В нашем случае сопротивления, учитывающие потери энергии на высшие гармоники, вошли в качестве составляющих в параметры $\Delta z_{\mathrm{d}}$ и $\Delta z_{\mathrm{q}}$.

Представим выражения для основной гармоники в матричной форме: 


\begin{tabular}{|c|c|c|c|c|c|c|}
\hline Impact Factor: & $\begin{array}{l}\text { ISRA (India) } \\
\text { ISI (Dubai, UAE } \\
\text { GIF (Australia) } \\
\text { JIF }\end{array}$ & $\begin{array}{l}=1.344 \\
=\mathbf{0 . 8 2 9} \\
=\mathbf{0 . 5 6 4} \\
=1.500\end{array}$ & $\begin{array}{l}\text { SIS (USA) } \\
\text { PИНЦ (Russia) } \\
\text { ESJI (KZ) } \\
\text { SJIF (Morocco) }\end{array}$ & $\begin{array}{l}=0.912 \\
=0.234 \\
=1.042 \\
=2.031\end{array}$ & $\begin{array}{l}\text { ICV (Poland) } \\
\text { PIF (India) } \\
\text { IBI (India) }\end{array}$ & $\begin{array}{l}=6.630 \\
=1.940 \\
=4.260\end{array}$ \\
\hline
\end{tabular}

$$
\left|\begin{array}{l}
\dot{U} \\
-j \cdot \dot{U}
\end{array}\right|=\left|\begin{array}{cc}
z_{d}+z_{1}^{+} & z_{d}+z_{1}^{-} \\
z_{q}+z_{1}^{+} & -\left(z_{q}+z_{1}^{-}\right)
\end{array}\right| \times\left|\begin{array}{c}
I_{1}^{+} \\
\dot{I_{1}^{-}}
\end{array}\right|
$$

Решим систему уравнений (9) для различных скольжений относительно параметров $I_{1}^{+}$и $I_{1}^{-}$, а также, определив $I_{\mathrm{d}}$ и $I_{\mathrm{q}}$, можно окончательно вычислить ток, потребляемый из сети:

$$
I=I_{d}+I_{q}
$$

В таком случае, потребляемая мощность будет рассчитана через выражение вида:

$$
P_{1}=U \cdot \operatorname{Re}(I)
$$

Коэффициент мощности можно будет выразить через модуль тока, потребляемого непосредственно из сети:

$$
\cos (\psi)=\frac{\operatorname{Re}(\dot{I})}{|\dot{I}|}
$$

где $|\dot{I}|$ - это модуль тока, потребляемого из сети.

В итоге, коэффициент полезного действия $\eta$ можно будет рассчитать по выражению вида:

$$
\eta=\frac{P_{2}}{P_{1}}
$$

Таким образом, использование эквивалентной однофазной машины при анализе асинхронного электродвигателя с экранированными полюсами позволяет достаточно просто вести расчеты основных рабочих характеристик и энергетических показателей двигателя.

Выводы, заключение. Приведенные зависимости для асинхронных двигателей с экранированными полюсами позволяют улучшить лишь некоторые рабочие и пусковые характеристики. Это означает, что подобные машины можно использовать намного эффективнее, с учетом того, что в последнее время все больше и больше выпускают двигатели малых мощностей.
Мы полагаем, что пусковые характеристики (а значит и рабочие) можно также улучшить путем применения из листов стали магнитных шунтов между полюсами, и путем увеличения воздушного зазора между неэкранированной частью полюсов, располагая на каждом полюсе несколько короткозамкнутых витков разной ширины.

Благодаря таким улучшениям, у асинхронных двигателей с экранированными полюсами повышается коэффициент полезного действия, и заметно уменьшаются энергетические потери.

С учетом того, что двигатели, которые используются в современных электробытовых приборах и автоматических устройствах однофазные, то и в быту, и промышленности, целесообразнее использовать однофазную сеть переменного тока.

С другой стороны, подобные двигатели имеют очень низкий крутящий момент, а значит, и небольшие размеры. Это позволяет создавать новые виды двигателей с несколькими витками, вообще без витков, но с неравномерным воздушным зазором. С учетом предложенных усовершенствований, пусковые и рабочие свойства двигателя можно заметно увеличить.

Улучшение предложенных характеристик позволит создавать инновационные конструкции двигателей, приспособленные к работам в специальных режимах, например, реверсивные, многоскоростные.

Таким образом, на сегодняшний день, многие вопросы, связанные с улучшением и усовершенствованием пусковых и рабочих характеристик асинхронных двигателей, разобраны очень детально и подробно, однако, математический аппарат, применяемый для описания этих процессов, остается довольно громоздким, а сами модели, используемые для анализа работы, требуют детальной наглядности в пространственно-временном масштабе (например, 3D-моделирование).

Поэтому, анализ однофазных асинхронных двигателей с целью совершенствования конструкции и повышения техникоэкономических показателей, остается актуальным, что требует дальнейших исследований и научных объяснений.

\section{References:}

1. Volynskij BA, Zejn EN, Shaternikov VE (1987) Jelektrotehnika. - Moscow: Izdatel'stvo "Jenergoatomizdat", 1987. - pp.424-426.
2. Mugalimov RG, Mugalimova AR, Mugalimova MR (2014) Tehnologija modernizacii tradicionnyh asinhronnyh dvigatelej na 


\begin{tabular}{|c|c|c|c|c|c|c|}
\hline Impact Factor: & $\begin{array}{l}\text { ISRA (India) } \\
\text { ISI (Dubai, UAE } \\
\text { GIF (Australia) } \\
\text { JIF }\end{array}$ & $\begin{array}{r}=1.344 \\
=0.829 \\
=0.564 \\
=1.500\end{array}$ & $\begin{array}{l}\text { SIS (USA) } \\
\text { PИНЦ (Russia } \\
\text { ESJI (KZ) } \\
\text { SJIF (Morocco }\end{array}$ & $\begin{aligned}= & 0.912 \\
= & 0.234 \\
= & \mathbf{1 . 0 4 2} \\
& =2.031\end{aligned}$ & $\begin{array}{l}\text { ICV (Poland) } \\
\text { PIF (India) } \\
\text { IBI (India) }\end{array}$ & $\begin{array}{l}=6.630 \\
=1.940 \\
=4.260\end{array}$ \\
\hline
\end{tabular}

jenergosberegajushhie asinhronnye dvigateli s individual'noj kompensaciej reaktivnoj moshhnosti. // Trudy VIII Mezhdunarodnoj (XIX Vserossijskoj) konferencii po avtomatizirovannomu jelektroprivodu AJeP2014. - Saransk: Izdatel'stvo "Nacional'nyj issledovatel'skij Mordovskij gosudarstvennyj universitet im. N.P. Ogarjova", 2014. - pp.211216.

3. Sugakov VG (2015) Vlijanie asimmetrii trehfaznoj pitajushhej sistemy na jelektrodvigateli peremennogo toka. // Vestnik Volzhskoj gosudarstvennoj akademii vodnogo transporta. - Nizhnij Novgorod: Izdatel'stvo "Volzhskij gosudarstvennyj universitet vodnogo transporta", 2015. - №44 (44). - pp.341-347.

4. Barabash AV, Perehodchenko VA (2016) Jelektrodvigateli peremennogo toka dlja shirokopolosnyh i tolstolistovyh stanov gorjachej prokatki. // Stal'. - M.: Izdatel'stvo "OOO Intermet Inzhiniring", 2016. - №2. pp.48-52.

5. Efimenko EI, Parojatnikov VM, Pogodin VN (2016) Odnofaznyj javnopoljusnyj jelektrodvigatel'. // Patent na izobretenie №2109390. - Patentnyj poisk, poisk patentov i izobretenij RF i SSSR. - Available: http://www.findpatent.ru/patent/210/2109390.ht $\underline{\mathrm{ml}}$ (Accessed: 13.07.2016).

6. Kacman MM (2002) Jelektricheskie mashiny avtomaticheskih ustrojstv. - Uchebnoe posobie dlja jelektrotehnicheskih special'nostej tehnikumov. - Glava 4.5. "Odnofaznye asinhronnye dvigateli $\mathrm{s}$ jekranirovannymi poljusami". - Moscow: Izdatel'stvo "Forum", 2002. - pp.77-79.

7. Baskutis PA (1957) Vrashhajushhij moment odnofaznogo asinhronnogo dvigatelja $\mathrm{s}$ jekranirujushhej obmotkoj. // Trudy Kaunasskogo politehnicheskogo instituta. Moscow, 1957. - Tom 1U. - pp.201-215.

8. Bukshnajtis IV, Katkjavichjus VI (1980) Vektornaja diagramma magnitnyh potokov statora asinhronnogo dvigatelja s jekranirovannymi poljusami. // Jelektrotehnika. - Moscow, 1980. - №3. - pp.12-14.
9. Marshak EL, Umancev RB (1974) Shemy obmotok mashin peremennogo toka. - Moscow: Izdatel'stvo "Jenergija", 1974. - 96 p.

10. Masandilov LB, Moskalenko VV (1968) Regulirovanie skorosti vrashhenija asinhronnyh dvigatelej. - Moscow: Izdatel'stvo "Jenergija", 1968. - $72 \mathrm{p}$

11. Larin AM, Guedidi Fauzi BK (2008) Jeksperimental'noe opredelenie parametrov shem zameshhenija sinhronnyh mashin pri predstavlenii obmotki vozbuzhdenija mnogokonturnoj cep'ju. // Jelektrotehnika i jelektromehanika. - Har'kov: Izdatel'stvo "Nacional'nyj tehnicheskij universitet Har'kovskij politehnicheskij institut", 2008. №3. - pp.37-40.

12. Sivokobylenko VF, Tkachenko SN (2012) Opredelenie parametrov shem zameshhenija glubokopaznyh asinhronnyh jelektrodvigatelej na osnove jeksperimental'nyh dannyh. // Jelektromehanicheskie i jenergosberegajushhie sistemy. - Kremenchug: Izdatel'stvo "Kremenchugskij nacional'nyj universitet im. Mihaila Ostrogradskogo", 2012. - №3 (19). pp.562-565.

13. Baranov EN, Stepanov AV, Tarasenko IA (2015) $\mathrm{K}$ raschetu perehodnyh processov $\mathrm{V}$ jelektricheskih cepjah s vzaimno induktivnymi svjazjami operativnym metodom. // Inzhenernyj vestnik. - Moscow: Izdatel'stvo "Moskovskij gosudarstvennyj tehnicheskij universitet im. N.Je. Baumana", 2015. - №11. - pp.12.

14. Vol'dek AI (1978) Jelektricheskie mashiny. Leningrad: Izdatel'stvo "Jenergija", 1978. - 832 p.

15. Korobov GV (1998) Issledovanie asinhronnyh dvigatelej s jekranirovannymi poljusami s cel'ju sovershenstvovanija konstrukcii. // Dissertacija na soiskanie uchenoj stepeni kandidata tehnicheskih nauk. - Special'nost' 05.09.01. "Jelektromehanika". - Voronezh, 1998. - pp.52.

16. Odilov G (2006) Magnitnoe pole v zazore i differencial'noe rassejanie obmotki faznogo rotora asinhronnogo dvigatelja. // Jelektrichestvo. - Moscow: Izdatel'stvo "Nacional'nyj issledovatel'skij universitet MJeI", 2006. - №6. - pp.42-47. 